\title{
Résistance variétale de la luzerne au nématode des tiges Ditylenchus dipsaci (Kühn) Filipjev; test d'évaluation et application en sélection
}

\author{
D Leclercq, G Caubel \\ INRA de Rennes, laboratoire de recherches de la chaire de zoologie, \\ domaine de la Motte-au-Vicomte, BP 29, 35650 Le Rheu, France
}

(Reçu le 20 novembre 1990; accepté le 24 avril 1991)

\begin{abstract}
Résumé - La résistance variétale à Ditylenchus dipsaci chez la luzerne est évaluée par un test de dépistage au stade plantule, fondé sur la caractérisation des symptômes exprimés après l'inoculation artificielle. La méthode employée présente certains facteurs de variabilité qui ont été étudiés. Ainsi, les symptômes à rechercher ont été précisés, les effets notateur et date de notation déterminés. II n'a pas été décelé de "pathotypes" de nématodes se multipliant sur les génotypes habituellement résistants. L'homogénéité des réponses des cultivars au test a été prouvée, et l'étude de la répétabilité a permis d'établir des références pour les variétés témoins, multiplicatrices et résistantes. L'application de la méthode à la sélection variétale a permis d'évaluer les niveaux de résistance de variétés étrangères et françaises, et donc de faire le point sur les sources actuelles de résistance génétique. Le suivi de variétés commerciales et de nombreux génotypes en cours d'amélioration a permis de démontrer qu'un cycle de sélection suffit pour élever le niveau de résistance d'un génotype multiplicateur du nématode au rang du témoin résistant. Quel que soit le niveau de sélection du génotype testé, la fonction "gain de résistance relatif" d'une génération à la suivante est une droite d'équation $Y=-0,64 X+54,5$.
\end{abstract}

luzerne / résistance variétale / nématode / Ditylenchus dipsaci / sélection

Summary - Varietal resistance of lucerne to the stem nematode Ditylenchus dipsaci (Kühn) Filipjev: the screening method and its application in selection for resistance. The current method used to estimate the varietal resistance of lucerne to the stem nematode Ditylenchus dipsaci is based on the characterisation of the symptoms the young plants exhibit after artificial inoculation. Several experiments have investigated these symptoms further; and differences have been found between observers' assessments (table $I V$ ) and the choice of scoring date (fig 1). Nematode pathotypes able to increase in resistant genotypes were not observed (fig 2, table III). The tested cultivars reacted homogeneously, as shown by results of 72 trials with the susceptible and resistant control varieties Europe and Vertus. The screening method allowed estimates to be obtained of the resistance of several varieties (fig 4, table V). Three varieties, Europe, Vertus and Cinna, and a great number of lucerne families were selected once or twice for resistance to the stem nematode (table VI). It is clear from this study that one simple selection is enough to make a susceptible cultivar as resistant as the resistant control variety Vertus. The gain (fig 3) in relative resistance $(Y)$ is given by the straight line relationship $\mathrm{Y}=-0.64 \mathrm{X}+54.5$ where $\mathrm{X}$ is the initial level of resistance (percentage of resistant plants).

lucerne / varietal resistance / stem nematode / Ditylenchus dipsaci / selection

\section{INTRODUCTION}

La création de variétés de légumineuses fourragères résistantes à Ditylenchus dipsaci constitue un objectif particulièrement intéressant pour intervenir contre ce parasite majeur. La résistance est caractérisée par la capacité du végétal à entraver la multiplication du nématode après pénétration dans la plante. Ce phénomène a été corrélé avec la réponse individuelle des plantes après l'inoculation contrôlée dans le cas de la luzerne, mais aussi du trèfle violet et même de la féverole. Ainsi, des méthodes d'estimation de la résistance, fondées sur la caractérisation des symptômes extériorisés précocément après l'inoculation artificielle de plantules, ont été proposées (Bingefors et Eriksson, 1968; Bingefors, 1971), discutées (Caubel, 1974; Caubel et al, 1977) et utilisées pour la création de variétés dans certains pays. La prise en compte de ce 
critère de sélection a conduit les sélectionneurs français, regroupés au sein de l'Association des créateurs de variétés fourragères (ACVF), à évaluer au laboratoire de nématologie de I'INRA de Rennes de nombreuses familles de luzerne et de trèfle violet (Caubel et al, 1985).

Afin d'améliorer la méthodologie et d'en étudier les limites dans une utilisation sur une grande échelle, des études ont été réalisées pour répondre aux nécessités de standardisation, de rapidité de mise en place, de facilité de lecture et de récupération des plantes à sélectionner. Cette publication vise tout d'abord à analyser la méthode d'estimation classiquement utilisée, puis à discuter les résultats obtenus sur l'évaluation, soit de variétés commerciales, soit de nombreuses familles en cours de sélection dans des établissements français et subissant éventuellement un ou plusieurs cycles de sélection pour la résistance à Ditylenchus dipsaci.

\section{MATÉRIEL ET MÉTHODES}

Les symptômes d'attaque de $D$ dipsaci observés sur luzerne 21-25 j après l'inoculation artificielle des plantules repiquées en rouleaux de papier buvard (Caubel et al, 1977) sont les suivants :

- le gonflement (G) : aspect boursouflé de l'hypocotyle, de l'épicotyle, des pétioles, décoloration et aspect satiné des tissus;

- l'arrêt de développment (A) : inhibition de la croissance terminale de l'apex: la plantule reste bloquée au stade cotylédonnaire ou unifolié.

Mais il est indispensable de bien caractériser les plantes :

- d'aspect sain (S) : la plante a repris son développement et sa croissance; elle a développé 1 ou 2 feuilles trifoliées mais reste de taille inférieure à un témoin non inoculé et présente des traces de pénétration des nématodes;

- non infectée (NI) : la plantule ne présente aucun symptôme, aucune trace de pénétration et se développe au même rythme qu'un témoin non inoculé.

Le test d'évaluation permet de déterminer pour chaque famille ou cultivar le pourcentage :

$G^{*}=100 \mathrm{G} /(T-\mathrm{M}-\mathrm{NI})$, rapport du nombre de plantes gonflées au nombre total diminué des plantes non infectées et manquantes.

Les caractéristiques des manipulations mises en place pour l'étude de la signification nématologique des symptômes, des facteurs de variabilité internes (choix de la date de notation, effet des populations de nématodes utilisées, influence du notateur) et entre plusieurs tests (homogénéité des cultivars testés, répétabilité des tests) sont reportées dans le tableau I.

\section{Signification nématologique et pertinence des symptômes}

L'étude conjointe des symptômes observés et des effectifs de nématodes correspondants est effectuée à la suite du test $A$.

\section{Étude des facteurs de variabilité}

\section{Date de notation : test A}

L'évolution des symptômes est observée globalement, avec des notations effectuées 13,25 et 32 j après l'inoculation. Chaque plantule est suivie individuellement.

\section{Populations de nématodes : tests $B$ et $C$}

L'étude comparative des populations est fondée sur le dénombrement des nématodes extraits des plantules (test B) et sur les symptômes extériorisés (test $C$ ). Les populations de $D$ dipsaci employées sont de diverses origines géographiques :

- L 102 et L 110 : populations de l'Orne, créées par croisements successifs à partir de couples individualisés;

- L 118 : population d'llie-et-Vilaine créée elle aussi par croisements successifs;

- FR : population naturelle de l'Orne;

- St Didier : population naturelle d'lle-et-Vilaine;

- Eriksson et SU : populations d'origine suédoise;

- DN : population d'origine danoise;

- Krusberg : population d'origine Nord-américaine.

Toutes ces populations sont élevées in vitro sur culture de cals de tissus (Bossis et Caubel, 1982), sauf St Didier.

\section{Notateur : test D}

Trois expérimentateurs ont effectué leur notation indépendamment les uns des autres avant de se regrouper pour une lecture commune.

\section{Homogénéité des cultivars testés : tests $\mathrm{H}$}

L'étude de l'homogénéité des réponses de cultivars et familles de luzerne est entreprise sur les résultats de 640 lots de semences soumis au test de résistance, soit un total d'environ 300000 plantules. Les effectifs des échantilions sont d'environ 500 plantules (les cas extrêmes étant 60 et 5600 individus), et 200 pour chaque témoin Europe et Vertus. Chaque rouleau de 20 plantules est considéré comme une répétition. 
Tableau I. Description des différentes expérimentations pour l'étude des facteurs de variabilité du test de résistance, des sources génétiques et des applications en sélection végétale.

\begin{tabular}{|c|c|c|c|c|c|c|c|c|}
\hline Test & Objectifs & Cultivars & $\begin{array}{l}\text { Effectifs } \\
\text { Plantes }\end{array}$ & $\begin{array}{l}\text { Populations Ir } \\
\text { Nématodes }\end{array}$ & Inoculum & $\begin{array}{l}\mathrm{T} \\
\left({ }^{\circ} \mathrm{C}\right)\end{array}$ & $\begin{array}{l}\text { Notation } \\
((1+n) \text { jours })\end{array}$ & $\begin{array}{l}\text { Durée } \\
\text { (mois) }\end{array}$ \\
\hline$A$ & Dates de notation & $\begin{array}{l}\text { Europe } \\
\text { Vertus }\end{array}$ & 400 & $\mathrm{DN}$ & 40 & 16 & $\begin{array}{l}13 \\
25\end{array}$ & 2 \\
\hline B & Populations de nématodes & $\begin{array}{l}\text { Europe } \\
\text { Vertus }\end{array}$ & 100 & $\begin{array}{l}\text { L } 102 \\
\text { L } 110 \\
\text { L } 118 \\
\text { Eriksson } \\
\text { Krusberg }\end{array}$ & 50 & $15-18$ & $\begin{array}{l}32 \\
80\end{array}$ & 3 \\
\hline C & Populations de nématodes & $\begin{array}{l}\text { Europe } \\
\text { Vertus } \\
\text { Cinna } \\
\text { Euver }\end{array}$ & 240 & $\begin{array}{l}\text { DN } \\
\text { FR } \\
\text { SU }\end{array}$ & 45 & $15-18$ & 25 & 1 \\
\hline $\mathrm{D}$ & Notateur (trois) & Euver & 315 & L 110 & 50 & $15-18$ & 21 & 1 \\
\hline$E$ & Variétés étrangères & $\begin{array}{l}12+ \\
\text { Europe } \\
\text { Vertus }\end{array}$ & 200 & $D N+F R$ & 40 & 16 & 25 & 1 \\
\hline$F$ & Variétés françaises & 23 & $300-500$ & St Didier & $30-45$ & 16 & 25 & 3 \\
\hline G & $\begin{array}{l}\text { Gains de résistance sur matériel } \\
\text { fixé }\end{array}$ & 15 & 360 & $\mathrm{DN}+\mathrm{FR}+\mathrm{SU}$ & U 35 & 16 & 25 & 1 \\
\hline $\mathrm{H}$ & Matériel en cours de sélection & $\begin{array}{l}498 \text { gén. } A C V \\
121 \text { Europe } \\
121 \text { Vertus }\end{array}$ & $\begin{array}{l}\text { F variable } \\
200 \\
200\end{array}$ & $\mathrm{DN}+\mathrm{FR}+\mathrm{SU}$ & $30-50$ & 16 & 25 & \\
\hline
\end{tabular}

\section{Répétabilité des tests : tests H}

La comparaison des tests entre eux est effectuée sur les 72 paires de témoins Europe et Vertus.

\section{Matériel végétal utilisé}

En présence des témoins Europe et Vertus, 12 variétés étrangères résistantes et 21 variétés inscrites au catalogue français sont évaluées au travers des tests $E$ et $F$. Les lots de semences proviennent des stations GEVES de La Minière et Du Magneraud, de l'INRA de Lusignan et de Montpellier, et de l'Association canadienne des producteurs de semences.

\section{L'étude des gains de résistance porte sur}

\section{du matériel fixe : test G}

L'expérimentation a pour objectif de comparer les niveaux de résistance acquis après 2 cycles de sélec- tion par les 3 variétés Europe, Vertus et Cinna. Le test d'évaluation confronte les 15 populations suivantes :

- les échantillons des variétés commerciales constituant les populations de départ $\mathrm{N}_{0}$;

- les descendances des plantes notées $A$ et $S$ au 1er test et conduites en autofécondation, constituant les 6 populations améliorées $\mathrm{N}_{1}$;

- les descendances des plantes des populations améliorées $N_{1}$, notées $A$ et $S$ au $2^{e}$ test et conduites en panmixie intrafamiliale, constituant les 6 populations améliorées $\mathrm{N}_{2}$.

La sélection a été réalisée à la station d'amélioration des plantes fourragères de l'INRA de Lusignan.

\section{du matériel en voie de sélection : tests $H$}

Des échantillons de semences de luzerne, correspondant à 498 familles fournies par l'association des créateurs de variétés fourragères (ACVF) ont été évalués et 1 à 2 cycles de sélection ont pu être réalisés dans les établissements pour 66 d'entre elles. 


\section{RÉSULTATS}

\section{Relation entre symptômes exprimés et nématodes dénombrés}

Le dénombrement des nématodes rencontrés dans les plantes 53 j après leur inoculation (tableau II) montre que les plantules notées $G$ présentent beaucoup de nématodes quel que soit le niveau de résistance du cultivar. Les plantules notées $A$ et $S$ ont peu ou pas de nématodes; de plus, les effectifs sont sensiblement plus élevés pour Europe que pour Vertus. Les 12 plantules de Vertus notées NI ne contiennent pas de nématodes; pour Europe, 3 plantules sur 8 en contiennent quelques-uns mais les 5 autres en sont exempts.

\section{Date de notation}

L'évolution des symptômes est exprimée en pourcentage de l'effectif total de chaque cultivar (fig 1). Pour Europe comme pour Vertus, si le taux de plantes gonflées augmente sensiblement entre $(I+13)$ et $(I+25)$ puis se stabilise à $(1+32)$, le taux de plantes en arrêt de développement diminue entre la première et la troisième notation, et fortement pour Vertus entre $(I+25)$ et $(I+32)$. Le taux de plantes d'apparence saine augmente fortement entre $(1+25)$ et $(1+32)$ chez Vertus, tandis que pour les pourcentages de plantes mortes et non infectées progressent peu chez les 2 variétés.

Par ailleurs, le suivi individuel des plantes Vertus fait apparaître une reprise importante de développement et de croissance des plantes en arrêt (32\% des effectifs notés $A$ deviennent $S$ ).
Pour Europe, ce transfert touche seulement $12 \%$ des $A$, au profit de $\mathrm{G}(30 \%)$ et $\mathrm{M}(19 \%)$.

\section{Populations de nématodes}

S'il n'apparaît pas de différence sensible entre la multiplication des populations pour Vertus (fig 2), ce n'est pas le cas pour Europe où la population Krusberg et, dans une moindre mesure, la population $\mathrm{L} 110$, se multiplient un peu moins bien que les autres.

Dans le test C, l'étude des symptômes fait apparaître des différences significatives entre les populations de nématodes, sans qu'il y ait d'interaction avec les cultivars. La population danoise $(66,5 \%$ de $G)$ se distingue des populations suédoise et française $(62,5 \%$ et $62,0 \%$ de G). Quant aux variétés, elles forment 3 groupes distincts (tableau III). Par ailleurs le pourcentage de plantes d'aspect sain est plus important pour la population de nématodes FR $(14,5 \%$ de $S)$ que pour DN $(6,0 \%)$ et SU $(3,8 \%)$.
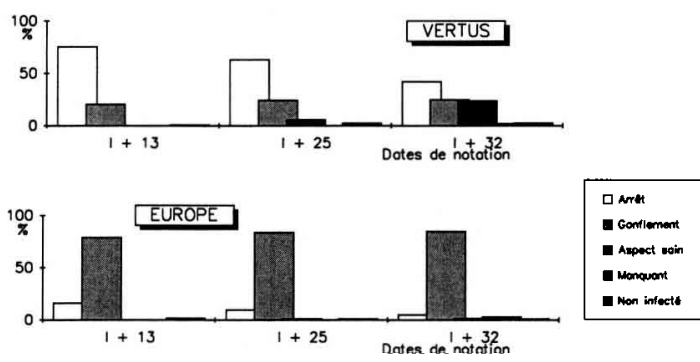

Fig 1. Évolution des symptômes chez Europe et Vertus en fonction de la date de notation, effectuée 13,25 et $32 \mathrm{j}$ après inoculation (test A).

Tableau II. Pourcentages des différentes catégories de symptômes 25 j après inoculation, et dénombrement des nématodes contenus dans les plantes $53 \mathrm{j}$ après inoculation (test $\mathrm{A}$ ).

\begin{tabular}{|c|c|c|c|c|c|c|}
\hline \multirow[t]{2}{*}{ Symptômes } & \multicolumn{3}{|c|}{ Europe } & \multicolumn{3}{|c|}{ Vertus } \\
\hline & $\%$ & Effectif moyen & Étendue & $\%$ & Effectif moyen & Étendue \\
\hline$A$ & 6 & 234 & $5-510$ & 43 & 64 & $0-180$ \\
\hline $\mathrm{G}$ & 85 & 2279 & $1150-3950$ & 26 & 2090 & $750-4100$ \\
\hline S & 3 & 244 & $0-630$ & 25 & 39 & $0-170$ \\
\hline $\mathrm{NI}$ & 2 & 0 & & 3 & 0 & \\
\hline
\end{tabular}




\section{Variabilité liée au notateur}

Des différences de notation s'observent dans $17 \%$ des cas (tableau IV). Elles concernent 9 fois sur 10 des plantules notées $A$ et $G$. Lorsque les plantes sont moins développées, donc extériorisent moins les symptômes (répétition 2), il y a plus de désaccords entre notateurs $(24 \%)$. Les divergences s'expriment différemment selon le notateur.

\section{Homogénéité des populations testées}

Afin de tester les écarts entre les résultats de chaque rouleau d'une même population de luzerne, des tableaux de contingence sont établis et un critère $2 \hat{~}$ (Arbonnier, 1966) est calculé. Si le critère 2 î reste inférieur à la valeur théorique correspondante extraite des tables du $\chi^{2}$ de Pearson, les différences entre rouleaux ne sont pas significatives donc la population a réagi de façon homogène à l'inoculation. Six cent trente sept populations sur 640 se trouvent dans ce cas. Toutefois, les critères 2 î des échantillons de Vertus sont supérieurs ou égaux, à effectifs équivalents, à ceux de Europe dans $80 \%$ des cas.

\section{Répétabilité des tests}

Malgré l'existence d'une variabilité non négligeable d'une expérimentation à l'autre, la comparaison des pourcentages de plantules gonflées sur les rouleaux témoins des 72 essais réalisés

Tableau III. Comparaison des moyennes (NewmanKeuls) et regroupement des cultivars et des populations de nématodes selon qu'ils se distinguent significativement ou non au seuil $\alpha=5 \%$ (test $C$ ).

\begin{tabular}{lllrl}
\hline D dipsaci & \multicolumn{2}{c}{ Gonflement } & \multicolumn{2}{c}{ Aspect sain } \\
& $\%$ & Groupe & $\%$ & Groupe \\
\hline & & & & \\
DN & 66,5 & a & 6,0 & b \\
SU & 62,5 & b & 3,8 & c \\
FR & 62,0 & b & 14,5 & a
\end{tabular}

\section{Cultivar}

\begin{tabular}{lllrl} 
Europe & 87,1 & $d$ & 5,0 & $f$ \\
Cinna & 65,0 & $e$ & 7,6 & ef \\
Euver & 63,5 & e & 8,9 & de \\
Vertus & 39,0 & $f$ & 10,6 & $d$ \\
\hline
\end{tabular}
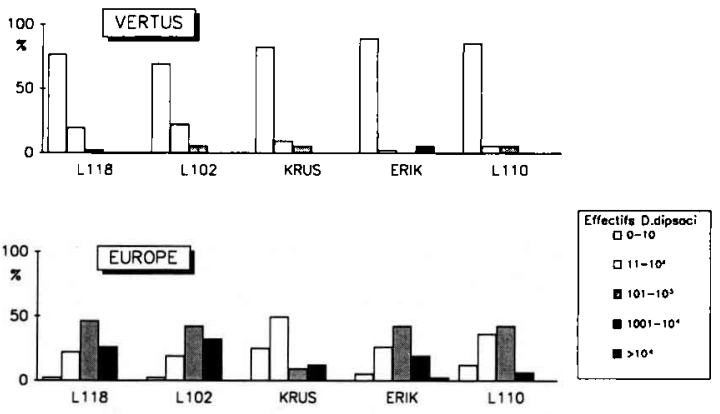

Fig 2. Pourcentages de plantes de chaque combinaison cultivar $\mathrm{x}$ population de nématode ( 30 plantes par combinaison) distribués selon les classes d'effectifs de nématodes contenus dans les plantes $80 \mathrm{j}$ après inoculation (test $\mathrm{B}$ ).

Tableau IV. Pourcentages du nombre de désaccords entre chacun des 3 notateurs et leur notation commune, et part de chacun dans l'erreur moyenne. Chaque terrine de 105 plantules constitue une répétition (test D).

\begin{tabular}{lllrr}
\hline & \% erreurs & not 1 & not 2 & not 3 \\
\hline Répétition 1 & 14,0 & 1,0 & 6,5 & 7,5 \\
Répétition 2 & 24,0 & 3,0 & 10,5 & 10,5 \\
Répétition 3 & 13,0 & 0 & 5,0 & 8,0 \\
Moyenne & 17,0 & 1,3 & 7,3 & 8,4 \\
\hline
\end{tabular}

entre 1987 et 1989 met en évidence l'homogénéité globale des notations effectuées sur Europe et Vertus. La comparaison des moyennes 2 à 2 par un test $t$ de Student fait ressortir que seuls quelques échantillons témoins ne sont pas représentatifs des valeurs moyennes de Europe $\left(84 \% \mathrm{G}^{*}\right)$ et Vertus $\left(32 \% \mathrm{G}^{*}\right)$ calculées sur l'ensemble des données, soient 5 couples EuropeVertus, 13 témoins Europe et 10 témoins Vertus; les essais correspondants sont donc écartés de l'étude ultérieure portant sur les génotypes ACVF.

\section{Sources de résistance génétique}

Parmi les 12 variétés étrangères signalées comme résistantes, 9 peuvent être classées dans les catégories "résistant", c'est-à-dire environ $30-45 \% \mathrm{G}^{*}$, et "assez résistant" c'est-àdire environ $45-60 \% \mathrm{G}^{*}$ (tableau V). 
Les variétés inscrites au catalogue français ont été éprouvées dans plusieurs tests séparés, réalisés dans des conditions parfois éloignées du "modèle» (tableau V). Une seule variété, Vertus, est classée "résistante»; Lunema, non inscrite au Catalogue est assez résistante; 7 variétés présentent un peu de résistance, les autres aucune.

\section{Gains de résistance}

\section{Matériel génétiquement fixé}

Un gain de résistance, exprimé en pourcentages de plantes $G^{*}, A^{*}$ et $S^{\star}$, est obtenu à chacun des cycles de sélection quel que soit le cultivar. II est d'autant moins marqué que la variété de départ est résistante (tableau VI). Ce gain ne diffère pas selon que les plantes sont sélectionnées sur les critères d'arrêt de développement ou d'apparence saine.

La comparaison des moyennes met en évidence l'homogénéité des résultats des populations améliorées suivant les critères $A$ ou $S$ à I'issue du fer cycle de sélection, quelle que soit la variété de départ. Quelques différences apparaissent après le $2^{\mathrm{e}}$ cycle chez Vertus où le gain de résistance est plus fort pour le critère $A$ et chez Cinna où ce gain est plus fort pour le critère $S$.

Les différences des niveaux de résistance des générations $N$ à $N+1$, rapportées au pourcentage $(A+S)^{*}$ de la génération $N$, permet d'établir la courbe de gain théorique de la sélection (fig $3)$. Sur ce tracé sont aussi reportés les résultats de la variété Alfa d'après Lundin (1969).

\section{Matériel en voie de sélection}

Les résultats obtenus sur la répétabilité des essais permettent de retenir 279 génotypes ACVF homogènes et comparable entre eux. La figure 4 met en évidence une proportion importante de génotypes $N_{0}$ non résistants au nématode puisque $57 \%$ de ceux-ci présentent plus de $70 \%$ de plantes gonflées. Il existe une variabilité importante parmi ces génotypes non sélectionnés et un nombre non négligeable de génotypes résistants. L'effet de la sélection est marqué car $76 \%$ des génotypes $N_{1}$ présentent moins de $40 \%$ de plantes gonflées.
De même, les résultats de 22 génotypes ayant subit 1 ou 2 cycles de sélection ont pu être superposés à la courbe de gain établie avec les résultats obtenus sur le matériel génétiquement fixé (fig 3).

Tableau V. Évaluation du niveau de résistance au nématode de quelques variétés étrangères test $E$, et de variétés inscrites au catalogue français test $F$. Pourcentages de plantes $\mathrm{G}^{*}$ et classement dans les catégories résistant (a), assez résistant (b), peu résistant (c) et non résistant (d). ( ${ }^{*}$ ) Le niveau de résistance de ces variétés est signalé à titre indicatif : il n'est pas connu avec précision. (**) Lunema n'a pas été admise au Catalogue.

\begin{tabular}{|c|c|c|c|}
\hline Cultivar & Origine & $\% G^{*}$ & Catégorie \\
\hline \multicolumn{4}{|l|}{ Test $E$} \\
\hline Vertus & Suède & 29,1 & $a$ \\
\hline Trek & Canada & 33,0 & $a$ \\
\hline Caliverde & Canada & 33,2 & $\mathrm{a}$ \\
\hline Otaio & Etats-Unis & 40,6 & $a$ \\
\hline Washoe & Etats-Unis & 41,5 & $a$ \\
\hline As $13 R_{+}$ & Etats-Unis & 41,5 & $\mathrm{a}$ \\
\hline Prescot & Etats-Unis & 52,0 & $b$ \\
\hline Blazer & Etats-Unis & 53,2 & $b$ \\
\hline Elevator & Etats-Unis & 55,0 & $\mathrm{~b}$ \\
\hline Vernema & Etats-Unis & 55,2 & $b$ \\
\hline Sparta & Etats-Unis & 61,0 & $b c$ \\
\hline Epic & Etats-Unis & 61,1 & bc \\
\hline Peack & Etats-Unis & 67,8 & c \\
\hline Europe & France & 80,8 & $d$ \\
\hline \multicolumn{4}{|l|}{ Test $F$} \\
\hline Vertus & Suède & 32 & a \\
\hline Lunema $\left({ }^{\star \star}\right)$ & Flamand & 51 & $b$ \\
\hline Medalfa & Provence & 61 & C \\
\hline Euver & Flamand & 62 & c \\
\hline Cinna & Provence & 62 & $c$ \\
\hline Polder & Marais & 63 & $\mathrm{c}$ \\
\hline Livia & Provence & $57^{\star}$ & $\mathrm{c}$ \\
\hline Alegro & Flamand & $68^{*}$ & $\mathrm{c}$ \\
\hline Lifeuil & Flamand & 69 & $\mathrm{~cd}$ \\
\hline Derby & Flamand & 72 & d \\
\hline Rival & Flamand & 76 & $d$ \\
\hline Resis & Flamand & 79 & d \\
\hline Europe & Flamand & 84 & d \\
\hline Hyb Milfeuil & Flamand & 84 & d \\
\hline Orchesienne & Flamand & $74^{*}$ & d \\
\hline Magali & Provence & $70^{*}$ & $d$ \\
\hline Dupuits & Flamand & $79^{\star}$ & d \\
\hline Belfeuil & Flamand & $76^{*}$ & d \\
\hline Lutece & Flamand & $85^{\star}$ & d \\
\hline Orca & Flamand & $79^{*}$ & $d$ \\
\hline Alize & Flamand & $73^{*}$ & $d$ \\
\hline Kara & Flamand & $76^{\star}$ & d \\
\hline Jade & Flamand & $73^{*}$ & d \\
\hline
\end{tabular}




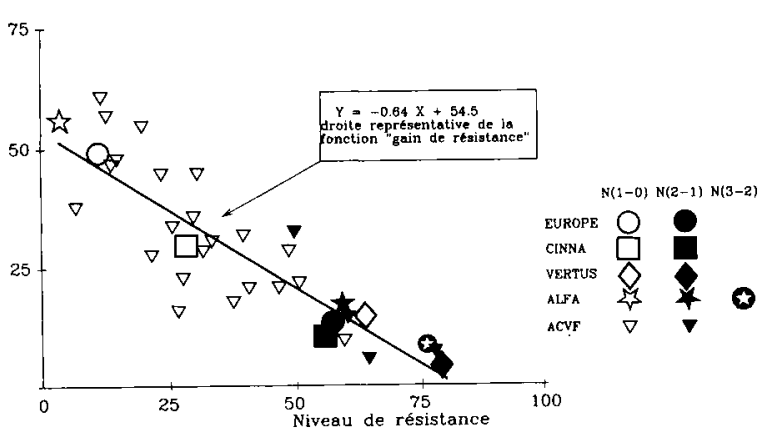

Fig 3. Gains de résistance $(Y)$ obtenus après un seul cycle de sélection, en fonction du niveau initial de résistance observé au départ $(X)$. La résistance est exprimée par le \% de plantes $(A+S)^{*}$, sur lesquelles porte la sélection. Données relatives au matériel fixé (test $G$ ), à la variété Alfa d'après Lundin (1969) et au matériel ACVF en voie d'amélioration (test $\mathrm{H}$ ), portant sur plusieurs cycles de sélection successifs et aboutissant à l'obtention des générations $N_{1}, N_{2}, N_{3}$ à partir du matériel $\mathrm{N}_{0}$. NB: L'équation de la droite de régression est calculée à partir des données sur Europe, Vertus, Cinna et Alpha.

\section{DISCUSSION ET CONCLUSION}

La relation qui apparaît entre le nombre de nématodes dans les plantules et le type de symptômes qu'elles extériorisent confirme les résultats antérieurs (Caubel, 1974). Les plantes présentant des gonflements (G) sont multiplicatrices de $D$ dipsaci. Par contre, les plantes en arrêt de développement (A) et d'aspect sain (S) ne sont pas multiplicatrices du nématode. Certes, le taux de multiplication ne semble pas complètement indépendant du niveau de résistance du cultivar. En effet, les effectifs de plantes $A$ et $S$ plus réduits pour Europe peuvent induire une erreur d'appréciation. En outre, pour un même phénotype, le génotype peut dépendre du niveau de résistance de la population (Guy, 1976). Enfin, les plantes notées NI n'ont pas été infectées. La végétation plus vigoureuse d'Europe augmente les risques d'erreur de notation, mais cette catégorie est suffisamment bien ca-

Tableau VI. Pourcentages de plantules notées $A, G$ et $S$ et appartenant à 3 variétés Europe $N_{0}, V_{e r t u s ~} N_{0}, C i n n a N_{0}$, et à leurs descendances respectives sélectionnées 1 (N1) et 2 fois (N2) pour la résistance à $D$ dipsaci sur les symptômes d'arrêt (A) ou d'aspect sain (S). Comparaison des moyennes (Newman-Keuls) et regroupement des cultivars selon qu'ils se distinguent significativement ou non au seuil $\alpha=5 \%$ (test $G$ ).

Gonflées

Population
Arrêtées

Population

Population
Saines

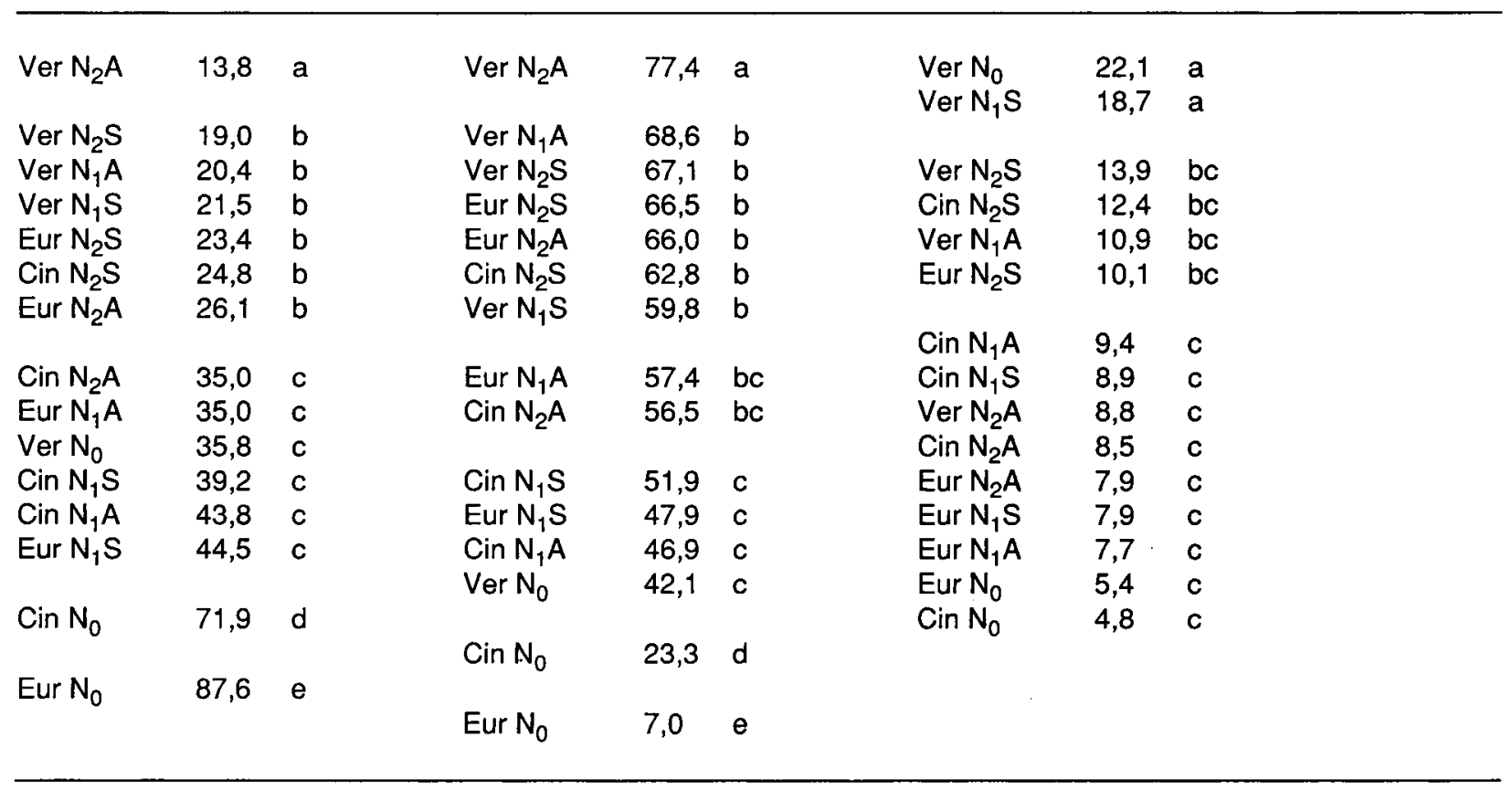




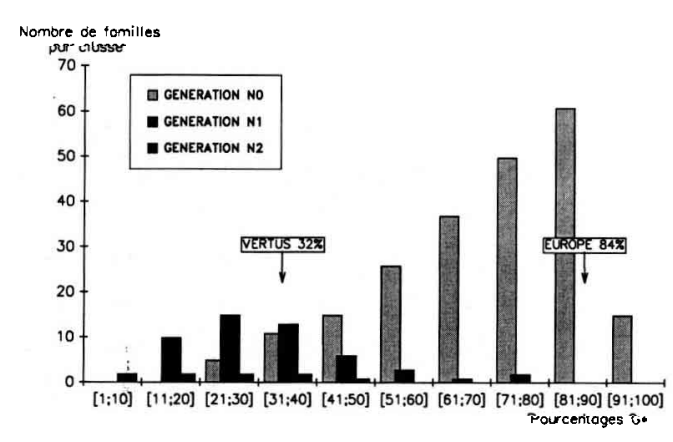

Fig 4. Distribution des familles de luzerne fournies par les sélectionneurs en fonction de leur pourcentage de plantes gonflées $G^{*}$. Distinction entre les familles non sélectionnées (génération $N_{0}$ ), et sélectionnées $1\left(N_{1}\right)$ ou 2 fois $\left(N_{2}\right)$ (test $H$ ).

ractérisée pour séparer les plantes d'aspect sain effectivement résistantes (S) de celles qui ont échappé à l'inoculation. La sélection de plantules non multiplicatrices passe donc par l'élimination des plantes gonflées (G) et non infectées (NI), et par le choix des plantes en arrêt de développement (A) et d'aspect sain (S).

Le symptôme le plus pertinent pour comparer les cultivars entre eux est le gonflement, seul symptôme de multiplication du nématode. Les types $A$ et $S$, plus sensibles à de faibles variations des conditions expérimentales, ne sont pas très pertinents, sauf si on en fait la somme, ce qui revient à utiliser la variable $1-\mathrm{G}$. Enfin, les critères $\mathrm{NI}$ et $\mathrm{M}$ essentiellement liés aux conditions expérimentales doivent être considérés comme des indicateurs de réussite ou d'échec des tests, mais jamais comme des estimateurs de résistance d'un cultivar.

Pour faire une estimation précise du niveau de résistance d'une population de luzerne, il faut, à une température de 15 à $17^{\circ} \mathrm{C}$, attendre $25 \mathrm{j}$ après l'inoculation. C'est en effet après 3 semains de culture que l'apparition des gonflements se stabilise. En sélection variétale, le respect de ce délai avant notation est fondamental car la proportion de plantules en arrêt de développement évoluant en plantules gonflées n'est pas négligeable pour les populations sensibles. Des symptômes tardifs, rares si les conditions expérimentales sont bonnes mais néanmoins inévitables dans un test précoce, peuvent apparaître les semaines suivantes. D'autre part, le suivi individuel des plantules justifie a posteriori l'utilisation en sélection variétale des plantes $S$, car elles apparaissent bien comme des plantes résistantes ayant repris précocément leur développement et leur croissance.
La luzerne réagit différemment en fonction des populations de $D$ dipsaci employées. Ces différences restent faibles en terme de symptômes, un peu plus marquées en terme de multiplication. L'étude du cycle de développement et des fécondités pourrait apporter des éléments de réponse quant à l'origine de ces différences. II convient donc, en sélection variétale, soit d'employer un inoculum composé d'un mélange de populations, soit d'utiliser la plus agressive. Mais s'il existe toujours, même pour un cultivar résistant, un certain pourcentage de plantes assurant la multiplication des quelques souches de nématode testées, il n'a pas été décelé comme Whitehead (1984) et Whitehead et al (1987), de populations capables de se multiplier sur Vertus. Cette divergence dans les résultats peut s'expliquer d'une part par le nombre réduit de souches étudiées, d'autre part par les conditions du test. II est dès lors nécessaire d'étudier l'évolution des populations de nématodes sur plusieurs générations successives en conditions naturelles, même si la tendance actuelle consiste à réduire la durée d'exploitation d'une luzernière à seulement 2 ans.

Les divergences liées au notateur s'expriment essentiellement sur les plantules peu développées, aux symptômes peu marqués. En respectant les conditions optimales de mise en place du test, donc en favorisant l'expression des symptômes, les risques de confusion diminuent sensiblement. L'expérience du notateur intervient bien évidemment dans le sens d'un meilleur discernement. La subjectivité du notateur joue aussi un rôle important, ainsi le notateur 2 est assez expérimenté, mais peu sévère, une deuxième notation devra intervenir plus tard. Par conséquent, une notation fiable réclame un réel apprentissage, mais aussi un contrôle périodique de la validité du jugement par des dénombrements de nématodes. Toutefois, un risque d'erreur de 1 à $3 \%$ semble difficilement compressible. En test de sélection variétale, le recours à un nombre réduit de notateurs formés conjointement limitera surtout les risques liés à la subjectivité.

Les rares cas d'hétérogénéité des populations testées ont pour cause un nombre insuffisant de rouleaux ou un résultat aberrant sur un rouleau. La variabilité plus forte observée chez Vertus par rapport à Europe s'explique par la difficulté à noter les cultivars résistants qui présentent un nombre non négligeable de symptômes intermédiaires (Caubel et al, 1977) dont le classement en $A$ ou $G$ pose problème. Mais la statistique du critère 2 î met en évidence l'homogénéité de ré- 
ponse de la quasi-totalité $(637 / 640)$ des populations de luzerne.

Concernant la répétabilité des tests, l'analyse met en évidence des différences d'un test à l'autre. Ces différences, dues à une extériorisation plus ou moins forte des symptômes, auraient pour origine certaines variations difficilement contrôlables de l'activité des nématodes, des conditions de culture, ou même de la pertinence du notateur. Dans quelques cas, ce sont un trop faible nombre de répétitions ou des rouleaux aberrants qui sont en cause. La répétabilité d'un test à l'autre n'est donc vérifiée que dans $60 \%$ des cas. Mais le nombre important de données expérimentales permet d'établir, pour Europe et Vertus une valeur de référence exprimée en pourcentage de plantes $G^{*}$ : pour Europe $84,4 \%$ $\pm 0,7 \%$ de $G^{*}$ calculé sur 9900 plantes et pour Vertus $32,1 \% \pm 0,9 \%$ de $G^{*}$ calculé sur 10160 plantes. II est dès lors possible de déterminer la validité d'un test de résistance en comparant statistiquement les résultats des rouleaux témoins aux valeurs moyennes de Europe et Vertus. Les familles testées dans des expérimentations différentes mais dont les témoins ne diffèrent pas des valeurs de référence établies précédemment deviennent ainsi directement comparables entre elles.

L'estimation de la résistance génétique de variétés étrangères, évaluées et sélectionnées par l'emploi de méthodologies différentes de la nôtre, met en évidence une concordance des résultats obtenus ici avec les niveaux de résistance signalés par ailleurs. Ils confirment aussi Vertus dans sa position de témoin résistant. D'autre part, plusieurs variétés du catalogue français, bien que non sélectionnées sur ce critère, présentent un pourcentage de plantes résistantes non négligeable, notamment dans le type Provence, et permettent de diversifier les sources de résistance. Mais l'obtention de nouvelles variétés plus résistantes et de bonne valeur agronomique reste un objectif des sélectionneurs.

En ce qui concerne les gains de résistance en sélection variétale, un cycle de sélection appliqué à une population multiplicatrice comme Europe suffit pour atteindre un niveau de résistance proche de celui de Vertus, et un deuxième cycle permet de le dépasser. II est donc possible d'améliorer rapidement le niveau de résistance de populations génétiquement fixées, ce qui apparaît comme techniquement et économiquement rentable. Les gains de résistance observés confirment les résultats obtenus par Bingefors
(1970) et Lundin et Jonsson (1975). L'effectif de la génération $N_{0}$, supérieur à 1000 plantes par variété, a permis de sélectionner des plantules à symptômes très nets, donc d'optimiser la réponse à la sélection; Lundin (1969) était parti d'un effectif de 25000 plantes!

Les différences entre les populations issues de notation $A$ et $S$ sont faibles ou nulles. Cet élément justifie une nouvelle fois l'utilisation de plantes notées $S$ en sélection variétale, car elles réagissent comme des plantes résistantes à reprise de développement précoce. Les différences observées sur la $3^{e}$ génération sont minimes et éventuellement liées à des effectifs parentaux plus réduits donc à une sélection sur des symptômes moins nets. Par ailleurs, le criblage selon $A$ ou $S$ semble induire une sélection secondaire sur la vitesse de reprise de développement puisque les populations issues de plantes $S$ en $2^{e}$ ou $3^{e}$ génération donnent plus de plantes d'aspect sain, et les populations issues de plantes $A$ donnent plus de plantes en arrêt de développement.

La courbe de gain constitue un modèle graphique qui permet d'estimer pour une population donnée le niveau de résistance potentiel, en fonction de celui de départ. Cette représentation des résultats fait apparaître une cohérence étroite entre les résultats enregistrés sur des variétés de niveaux de résistance très différents au départ. Ces données et celles obtenues par Lundin (1969) avec la variété Alfa testée sur 4 générations permettent d'établir une droite de régression négative d'équation $Y=-0,64 X+54,5$ avec un coefficient de corrélation $r=-0,98$ très significatif (fig 3 ). En outre, cette équation permet d'estimer le nombre de cycles nécessaires pour atteindre un niveau de résistance donné.

Les résultats obtenus sur des populations et lignées de luzerne en cours de sélection confirment ceux obtenus sur quelques variétés connues. En un cycle de sélection, il est ainsi possible d'atteindre, voire de dépasser le niveau du témoin résistant Vertus. Dans tous les cas, l'accroissement du pourcentage de plantes résistantes après un cycle de sélection diminue d'autant plus que le génotype de départ est résistant. En ce qui concerne les gains de résistance relatifs, les points observés après 1 ou 2 cycles de sélection, s'inscrivent de façon assez homogène autour de la droite précédemment définie. Les rares résultats très éloignés correspondent à des conditions limites; ou bien le nombre de plantes est trop faible, ou surtout les 
taux de plantes mortes ou non infectées sont plus élevés que la normale, en raison d'une qualité médiocre de l'inoculum ou d'incidents de température. II est donc essentiel de respecter, autant que possible, l'ensemble des conditions de mise en place du test.

En conclusion, la méthode d'estimation de la résistance réalisée sur des plantules apparaît comme fiable et utilisable pour des objectifs de caractérisation et de sélection. Elle permet par ailleurs des gains rapides de niveau de résistance dès le premier cycle de sélection. Toutefois, le travail de sélection étant conduit sur des populations de luzerne, la résistance obtenue ne peut être que partielle, avec présence d'un certain pourcentage, même faible, de plantes multiplicatrices du nématode. II conviendra donc d'étudier ultérieurement ce qu'il advient de cette résistance dans les conditions agronomiques, en particulier l'impact de petits foyers multiplicateurs sur le développement éventuel d'attaques dans la luzernière.

\section{REMERCIEMENTS}

Ce travail, réalisé au laboratoire de zoologie de l'INRA Le Rheu a été financé en partie par le ministère de l'Agriculture (de 1985 à 1989) dans le cadre du contrat INRA-Association des créateurs de variétés fourragères.

Nos remerciements s'adressent aux sélectionneurs participant au programme (GIE Amélioration fourragère, Blondeau, Florimond Desprez, Green Genetics, INRA Lusignan, Lafite, Tourneur grandes cultures obtention. UNCAC Serasem, Verneuil recherche), aux deux secrétaires successifs de l'ACVF, MM Charpentier et Gayraud ainsi qu'aux chercheurs étrangers qui nous ont fournis nématodes et semences. Nous remercions tout particulièrement les établissements Desprez, Tourneur, et INRA Lusignan qui nous ont permis l'accès à leurs sélections, et nos collègues de I'INRA DGAP de Lusignan, Mme Procheron et MM Ecalle Genier et Guy pour leur collaboration constante au cours de la réalisation de ce travail.

\section{RÉFÉRENCES}

Arbonnier P (1966) L'analyse de l'information : aperçu théorique et application à la loi multinomiale. Ann Sci For 23, 950-1017

Bingefors S (1970) Resistance against stem nematodes Ditylenchus dipsaci (Kühn). Filipjev EPPO Publ 54, 63-75

Bingefors S (1971) Resistance to nematodes and the possible value of inducted mutations. Agence Int Energ At Bull 209-235

Bingefors S, Eriksson KB (1968) Some problems connected with resistance breeding against stem nematodes in Sweden. Z Pflanzenzuecht 59, 359. 375

Bossis M, Caubel G (1982) Elevage monoxénique de nématodes phytoparasites sur cals de tissus. $\mathrm{Sci}$ Agron Rennes 115-124

Caubel G (1974) Réactions de 3 variétés de luzerne à l'inoculation des plantules par le nématode des tiges Ditylenchus dipsaci. Sci Agron Rennes 37-42

Caubel G, Bossis M, Genier G, Guy P (1977) Mise au point d'un test de sélection de luzernes résistantes à Ditylenchus dipsaci. Sci Agron Rennes 25-32

Caubel G, Charpentier F, Guy P (1985) Résistance variétale des Légumineuses Fourragères à Ditylenchus dipsaci (Kühn) Filipjev. CR Séances Acad Agric France 7, 719-729

Guy P (1976) Déterminisme génétique de la résistance à Colletotrichum trifolii chez Medicago sativa L. Ann Amélior Plant 26, 215-234

Lundin P (1969) Breeding of lucerne for resistance to stem nematode and Verticillium wilt. Sver Utsädesfören Tidskr 79, 133-137

Lundin P, Jonsson HA (1975) Weibulls'Vertus, a lucerne variety with high resistance to stem nematode and Verticillium wilt. Agric Hortique Genet 33, 17-32

Whitehead AG (1984) Interaction of three lucerne cultivars and eleven English isolates of stem nematode (Ditylenchus dipsaci) "lucerne race". Plant Pathol 33, 33-37

Whitehead AG, Janet E, Fraser, Nichols AJF (1987) Variation in the development of stem nematode ( $D i$ tylenchus dipsaci) in susceptible and resistant crop plants. Ann App/ Biol 111, 373-383 